\title{
Surface engineering of biaxial Gd2Zr2O7 thin films deposited on Ni-5at\%W substrates by a chemical solution method
}

Yue, Zhao; Grivel, Jean-Claude; Liu, Min; Suo, Hongli

Published in:

CrystEngComm

Link to article, DOI:

10.1039/c2ce06613k

Publication date:

2012

Document Version

Publisher's PDF, also known as Version of record

Link back to DTU Orbit

Citation (APA):

Yue, Z., Grivel, J-C., Liu, M., \& Suo, H. (2012). Surface engineering of biaxial $\mathrm{Gd}_{\mathrm{Zr}} \mathrm{Zr}_{\mathrm{O}}$ thin films deposited on $\mathrm{Ni}-5$ at\%W substrates by a chemical solution method. CrystEngComm, 14(9), 3089-3095.

https://doi.org/10.1039/c2ce06613k

\section{General rights}

Copyright and moral rights for the publications made accessible in the public portal are retained by the authors and/or other copyright owners and it is a condition of accessing publications that users recognise and abide by the legal requirements associated with these rights.

- Users may download and print one copy of any publication from the public portal for the purpose of private study or research.

- You may not further distribute the material or use it for any profit-making activity or commercial gain

- You may freely distribute the URL identifying the publication in the public portal 


\title{
Surface engineering of biaxial $\mathrm{Gd}_{2} \mathrm{Zr}_{2} \mathrm{O}_{7}$ thin films deposited on $\mathrm{Ni}-5 \mathrm{at} \% \mathrm{~W}$ substrates by a chemical solution method
}

\author{
Yue Zhao, ${ }^{* a}$ Jean-Claude Grivel, ${ }^{a}$ Min Liu ${ }^{b}$ and Hongli Suo ${ }^{b}$ \\ Received 1st December 2011, Accepted 17th January 2012 \\ DOI: 10.1039/c2ce06613k
}

\begin{abstract}
The surface texture and morphology of thin films play an essential role in determining their properties. In this study, local features in the film surface of crystallized $\mathrm{Gd}_{2} \mathrm{Zr}_{2} \mathrm{O}_{7}(\mathrm{GZO})$ films with a thickness gradient are investigated by means of scanning electron microscopy and electron backscatter diffraction. A strong dependence of the morphology and texture on the film thickness is observed, mainly due to (i) the transition of growth mode associated with the critical film thickness, i.e., increasing the film thickness leads to the grain morphology changing from 2-dimensional discs (highly ordered nanoislands or network) to 3-dimensional domes (equiaxed nanograins), and (ii) the segregation of residual carbon in the surface layer. The epitaxial nuclei forming at the interface hardly further develop by consuming the polycrystalline grains in the surface layer. A two-step annealing procedure is proposed to improve the surface texture in the $\mathrm{GZO} / \mathrm{NiW}$ system, according to the influence of $\mathrm{PO}_{2}$ on the texture formation of GZO films. A GZO film with strong biaxial texture is achieved, as evidenced by the high indexing rate of Kikuchi patterns on the film surface, as well as by the highly ordered crystal structure along the film thickness observed by a transmission electron microscope. On the basis of the enhanced understanding of the crystallization processes, we demonstrate a possibility of engineering the surface morphology and texture in the film deposited on technical substrates using a chemical solution deposition route.
\end{abstract}

\section{Introduction}

The crystallographic texture and defined nanostructure of thin films have been receiving great interest because of their essential role in determining a large range of structural and functional performances, such as wear resistance, electronic, magnetic, optical and superconducting properties. ${ }^{1-3}$ Among all these fields of research, the production of long lengths of coated conductors $\left(\mathrm{REBa}_{2} \mathrm{Cu}_{3} \mathrm{O}_{7-x}, \mathrm{RE}=\mathrm{Y}\right.$ and rare earth $)$ with potential applications is a successful case, which is achieved through deposition of multilayer structures based on epitaxial growth on a textured substrate. In order to achieve high critical current densities, $J_{\mathrm{c}}>$ $1 \mathrm{MA} \mathrm{cm}^{-2}$ in the superconducting films, a good crystallographic alignment needs to be transferred to the superconductor from epitaxial oxide buffer layers (such as $\mathrm{CeO}_{2}, \mathrm{LaMnO}_{3}$, etc.) deposited either on rolling assisted biaxially textured substrates (RABiTS) or by means of ion beam assisted deposition techniques on untextured templates. ${ }^{4-6}$ On the other hand, control of the morphology and nanostructure in buffered substrates has also attracted more and more attention. Such alternative approaches to enhance the superconducting performance include surface sulfurization, ${ }^{7}$ surface decoration, ${ }^{\mathbf{8}, 9}$ surface atomic

${ }^{a}$ Materials Research Division, Risø National Laboratory for Sustainable Energy, Technical University of Denmark, Frederiksborgvej 399, DK-4000 Roskilde, Denmark. E-mail: zhyu@risoe.dtu.dk

${ }^{b}$ Institute of Materials Science and Engineering, Beijing University of Technology, Beijing 100124, China flattening, ${ }^{10,11}$ surface morphology modulation, ${ }^{12-14}$ etc. Lattice mismatch, chemical and thermal stability, homogeneity, texture, crystallinity and roughness play essential roles in optimising the superconducting performance of the $\mathrm{REBa}_{2} \mathrm{Cu}_{3} \mathrm{O}_{7-x}$ layer grown subsequently on top of the buffer layer stack.

The chemical solution deposition route, being a bottom-up deposition technique, has many advantages, e.g., highthroughput manufacture with low cost, and ability of tuning the self-assembled nanostructures by solution chemistry and crystallization processes. ${ }^{15-17}$ These are the two reasons explaining why such a technique has been extensively employed in fabricating a broad spectrum of oxide films with unique properties. However, in contrast to the processes used for other functional oxide films deposited generally on single crystal substrates in an oxidizing atmosphere, a reducing/inert atmosphere has to be used during film crystallization in the case of superconducting coated conductors to minimize film-substrate reaction and metal surface oxidation when using technical metallic substrates for practical applications. Therefore, the effect of the atmosphere on the pyrolysis and crystallization processes as well as on the substrate has to be taken into consideration. Recently, it has been already realized that minor atmosphere differences (humidity, oxygen partial pressure, flow rate, etc.) during film preparation have a strong influence on structural features, such as homogeneity, morphology, texture, etc. ${ }^{18-22}$ and thus on their performance. More knowledge on the atmosphere effect in particular on the surface quality of films derived from chemical 
solutions is still needed in order to manufacture homogeneous long lengths of multilayer structures comprising ceramic oxides on a metallic substrate. Moreover, due to its complexity, the mechanism of nucleation and growth in the amorphous film starting from a wet precursor is also not clear so far. Full understanding of the aforementioned issues will facilitate the development and application of oxide thin films in general.

The methodology presented in this work is based on the study of a film with a thickness gradient, which provides plenty of information with respect to the morphology development related to the growth mode transition, as well as texture formation. Carbon residue in crystallized films, a general issue for chemical solution methods, is also discussed in detail. We herein selected $\mathrm{Gd}_{2} \mathrm{Zr}_{2} \mathrm{O}_{7}(\mathrm{GZO})$ films grown on $\mathrm{Ni}-5 \mathrm{at} \% \mathrm{~W}$ substrates $(\mathrm{NiW})$ as a model system, because GZO is one representative material with pyrochlore structure, which can be used for a wide variety of technological applications, such as ionic conductor in solid-oxide fuel cells, and components in the nuclear fuel cycle. ${ }^{23} \mathrm{GZO}$ also is considered as a promising buffer layer material in coated conductors with a simplified configuration, thanks to the compromised lattice mismatch between the superconducting layer and the technical substrate underneath and excellent barrier behavior against oxygen diffusion. ${ }^{24,25}$

\section{Experimental procedure}

\section{Film preparation}

The GZO films were produced by a standard MOD route, including solution synthesis, dip coating and crystallization. ${ }^{26}$ The total cation concentration of the GZO precursor solution and the withdrawal speed for coating the wet films are fixed at $0.6 \mathrm{~mol} \mathrm{~L}^{-1}$, and $10 \mathrm{~mm} \mathrm{~min}^{-1}$, respectively. A film thickness of about $30 \mathrm{~nm}$ is obtained in a homogeneous zone under these conditions. A textured NiW alloy tape (Evico $\mathrm{GmbH}$ ) is used as a substrate. Before coating, the bare NiW alloy tape is annealed in a flowing $5 \% \mathrm{H}_{2} / \mathrm{Ar}$ gas at $850{ }^{\circ} \mathrm{C}$ for $20 \mathrm{~min}$ to remove the thin oxide layer formed during storage. Crystallization annealing is performed in the heat treatment system with an $\mathrm{O}_{2}$ probe (Australian Oxytrol Systems Pty. Ltd), where both the $\mathrm{O}_{2}$ and the temperature close to the sample are monitored online during the whole heat treatment processes. A broad range of $\mathrm{PO}_{2}$ (from $1.3 \times 10^{-19}$ atm to $1 \%$ ) in the annealing atmosphere can be reached by adjusting the flow ratio of $5 \% \mathrm{H}_{2} / \mathrm{N}_{2}$ and $1 \% \mathrm{O}_{2} / \mathrm{Ar}$ forming gases. The $\mathrm{PO}_{2}$ values are calculated from the corresponding potential values readout from the multimeter connected to the probe according to the Nernst equation. Two types of heat treatment profiles are used for crystallizing GZO precursor films. For the conventional annealing process, the precursor film is crystallized at $1000^{\circ} \mathrm{C}$ for $60 \mathrm{~min}$ in the atmosphere with a constant $\mathrm{PO}_{2}$. In order to enhance the grain growth, we also proposed a two-step annealing process, where a conventional annealing section with shorter dwell time (i.e., 30 min) under a low $\mathrm{PO}_{2}$ atmosphere is followed by a 30 min dwelling under a higher $\mathrm{PO}_{2}$ atmosphere at the same temperature.

\section{Characterization}

The phase content and texture of the films were investigated by a four-circle diffractometer (Bruker D8) using $\mathrm{Cu} \mathrm{K} \alpha$ radiation. The reflections (222) and (004) of GZO were used to characterize the in-plane and out-of plane textures in GZO/NiW samples. Electron backscattering diffraction (EBSD) measurements for local surface texture analysis in local were carried out in a scanning electron microscope (SEM, from Zeiss Supra 35) equipped with a HKL detector. The Kikuchi patterns were automatically analyzed by the data handling software package (Channel 5). The surface morphology of the films was observed by a DualScope atomic force microscope (DME Danish Micro Engineering A/S, Denmark) with a silicon tip in tapping mode and the SEM with an in-lens detector.

X-Ray photoelectron spectroscopy (XPS) measurements were conducted on the films surface in a Thermo Scientific K-Alpha $\mathrm{X}$-ray Photoelectron Spectrometer system using mono-chromatic $\mathrm{Al} \mathrm{K} \alpha$ radiation $(1486.7 \mathrm{eV}$ ) with a beam size of about 0.4 $\times 0.4 \mathrm{~mm}^{2}$. The pressure in the analyzer chamber was around $10^{-8} \mathrm{~Pa}$ during the measurements. To acquire the element depth profiles, a sputtering source providing $\mathrm{Ar}^{+}$ions was used. The etch speed mentioned in this study corresponds to a sputtering rate of about $1 \mathrm{~nm} \mathrm{~min}{ }^{-1}$ on Ta oxide. A transmission electron microscope (TEM) cross-sectional sample was prepared by conventional mechanical polishing and grinding followed by ion milling using a Gatan691 thinning machine operating under the voltage reducing from $4.8 \mathrm{kV}$ to $3.2 \mathrm{kV}$ gradually for $30 \mathrm{~min}$. The angle for the ion milling process was also decreased from $15^{\circ}$ to $4^{\circ}$ during the voltage descent. TEM observations were performed using a Tecnai G2 F20 S-Twin operating at $200 \mathrm{kV}$.

\section{Results and discussion}

\section{Morphology dependence on film thickness}

Film thickness doubtlessly plays a critical role in film structure and performance no matter which deposition technique is used. Nonetheless, investigation of the thickness effect on metallic substrates is not an easy issue, because of (i) the complexity of the substrate (grain boundary grooving as morphology variation and misorientation from grain to grain as texture variation) and (ii) the high sensitivity of the solution deposition technique (solution chemistry and processing conditions uncertainty). Taking advantage of the dip coating technique, a region with thickness gradient in the wet film can be obtained around the boundary of the bare substrate and the film. This film thickness variation feature is still present in the crystallized film. Considering the relatively large grain size of the NiW substrate underneath, entire areas with GZO nanosize grains are grown on individual NiW grains, providing information about the variation of crystallization behavior only associated with film thickness. The effect of the other variables (i.e., substrate variation, processing atmosphere, etc.) can in principle be neglected.

As shown in Fig. 1a, the morphology in the region on an individual NiW grain changes from a partially covered nanoisland structure to a fully covered film with nanosize pinholes. Three zones with different morphological features, although without clear boundaries, can also be distinguished in the partially covered region (Fig. 1b). In zone I, nanodots with grain size smaller than $20 \mathrm{~nm}$ are completely isolated, and most often the nanoislands with rectangular shape are geometrically well aligned with respect to the orientation of the substrate underneath, i.e. the edges of the rectangle are parallel to the rolling 

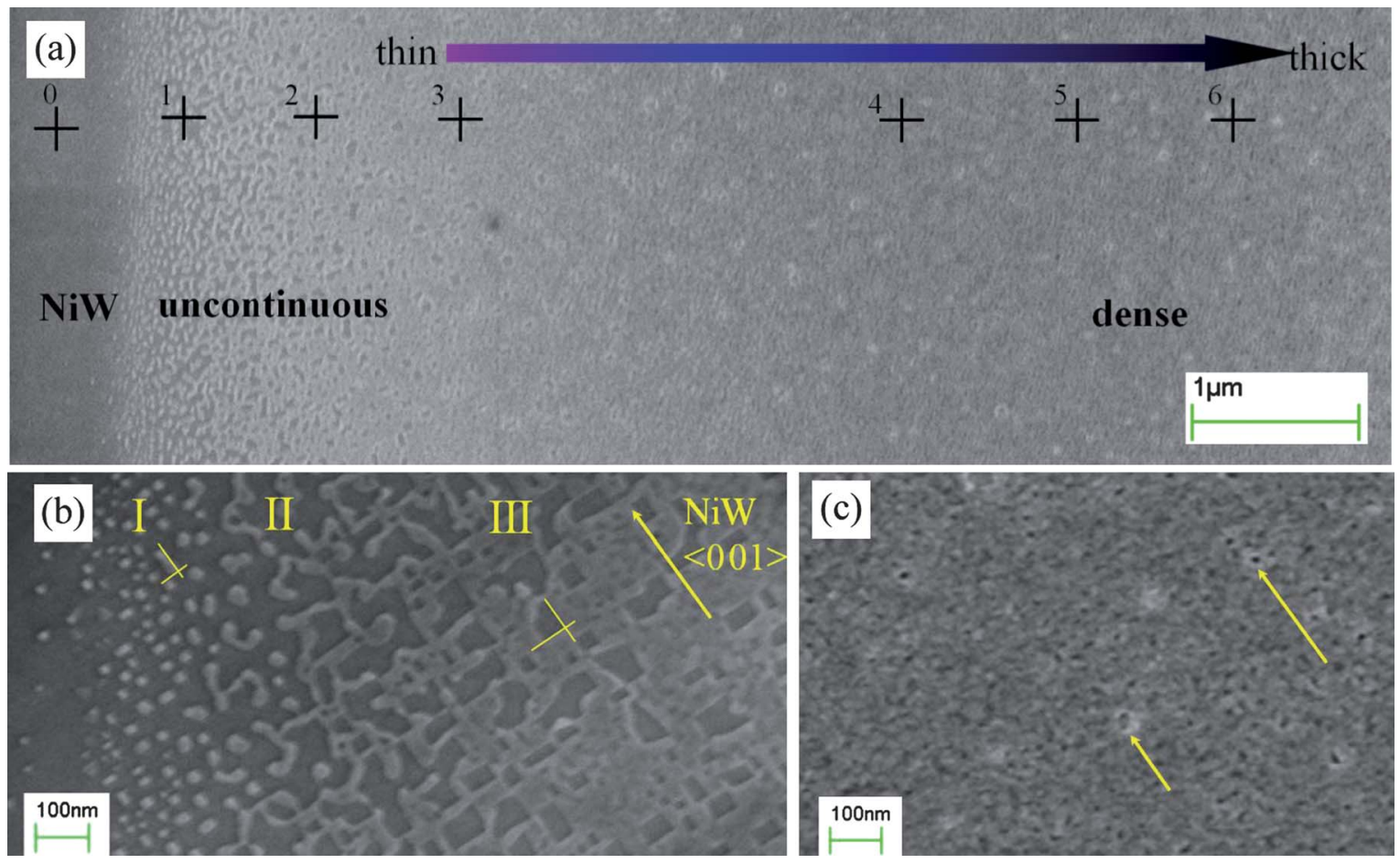

Fig. 1 (a) Panorama of the film with thickness gradient on an individual NiW grain. (b) Zoom in image of uncontinuous region with three zones. The edge of a nanograin in zone I and that of a nanohole in zone III are marked, while the NiW $\langle 001\rangle$ direction of the substrate is shown by the arrow. (c) Zoom in image of the fully covered region, corresponding to the right part in (a). Note that the film was coated with the withdrawal speed of 10 mm $\min ^{-1}$, followed by crystallization at $1000{ }^{\circ} \mathrm{C}$ for $30 \mathrm{~min}$ in a strongly reducing atmosphere.

direction (or transverse direction) of the substrate. In zone II, the nanoislands start to agglomerate and larger grains with irregular shapes are observed. The semi-network of covered region forming in this zone could be considered as a metastable configuration. Regulating either the crystallization temperature or the dwell time might change the grain shape and configuration in this zone. In zone III, plate-like grains with a large ratio of lateral grain size to thickness exhibit a high degree of flattening on the top surface, and they form a connected network with incorporated nanoholes. The nanoholes in zone III show a similar geometric orientation with respect to the substrate as the rectangular grains in zone I. When further increasing the film thickness, we obtain a nearly or completely fully covered region, where the grain size starts to decrease and remains constant at about $10 \mathrm{~nm}$. In the fully covered region, the typical morphology of solution derived films is present, which is almost thickness independent. Equiaxed nanograins with dome-like top surface and irregular shaped pinholes are two main features (Fig. 1c).

In general, the nucleation and growth of films derived from chemical solution routes involves a transition from amorphous to crystalline state. Contrary to other physical deposition techniques, one of the characteristics for a chemical solution route is that it is an ex situ process without continuous adatom attachment taking place during crystallization. Therefore, the region with thickness gradient is a unique example to study the microstructure variations in the film. In the following, we will discuss the origin of the microstructure formation following changes of two characteristic features during the morphology evolution: grain size and shape. Comparing zone I and II, it is found that the increase in the grain size is accompanied by a decrease in the density of the isolated nanograin. This is due to the higher nuclear density when increasing the amount of the materials in this thicker region (from I to II). As soon as the distance between nuclei decreases to a level comparable to the surface diffusion length, the mass transport/redistribution between the isolated grains could drive the crystalline growth to minimize surface energy. ${ }^{27}$ On the other hand, according to the crystallographic orientation of these nanograins (which will be discussed in detail in Section 3.2), the geometrical alignment of the GZO crystallites in zones I and III can be described as: GZO (002) facet//NiW (002) as substrate surface, while $\mathrm{GZO}\langle 110\rangle$ as edge//NiW $\langle 001\rangle$ as transverse or rolling direction of the substrate. These geometrical alignments are consistent with the epitaxial relationship between the GZO crystalline and the NiW substrate, therefore, indicating that the development of the grain shape in the equilibrium configuration is highly restricted by crystallographic orientation of the substrate. The morphology configuration in these regions implies that the film-substrate interface energy (surface and interface elastic energies) is dominating. In the fully covered region, the lateral grain size turns to become much smaller (reduced to about $10 \mathrm{~nm}$ ), and the geometric alignment of the crystallites (or of the holes) disappears. Additionally, the grains in this region exhibit a convex top surface instead. Both the grain size and the shape changes could be explained by the classic growth mechanism, ${ }^{28}$ i.e., the interaction strength between GZO adatoms and the surface of NiW substrate. When GZO adatoms attach preferentially to NiW surface sites, two dimensional growth, i.e. Frank-van der Merwe growth $(\mathrm{FW})$, dominates, leading to formation of grains with an atomically smooth top. Conversely, when GZO adatom-adatom 
interactions are stronger than those of the GZO adatom with the NiW surface, the GZO crystallites form preferentially threedimensional clusters, i.e., Volmer-Weber growth mode (VW). It is known that the transition from FW to VM mode occurs at a critical thickness. Thus the observation of crystallites with convex top surface in the dense region is an indication of growth mode transition. This is also in agreement with the trend of the grain size change. As a conclusion, the morphology in this region with thickness gradient is determined not only by the material amount in the precursor layers (i.e. film thickness), but also by the existence of a critical thickness that is highly dependent on chemical and physical properties (surface energies and lattice parameters). The significance of this study lies in the understanding of the morphology dependence on film thickness, as well as on offering an idea to manipulate the morphology of thin films with defined nanostructures by regulating the processing conditions and the properties of the film-substrate interface.

\section{Surface texture dependence on film thickness}

Not only the morphology but also the surface texture show a strong dependence on film thickness, which will be discussed as follows according to EBSD results. Kikuchi patterns at the points numbered from 0 to 6 in Fig. 1a are shown in Fig. 2. As expected, a typical Kikuchi pattern representing textured NiW grains is obtained in the bare substrate (position 0), while another one due to $\mathrm{GZO}$ grains with $45^{\circ}$ rotated orientation with respect to the underlying $\mathrm{NiW}$ grain is obtained at positions 2 and 3 roughly corresponding to zone III shown in Fig. 1b. Two sets of patterns including strong reflections of $(\overline{2} \overline{2} 0) \mathrm{Ni}$ and $(\overline{2} \overline{2} 0)$ GZO coexist in the nanoisland zone. As the film thickness further increases (from position 4 to 6), the Kikuchi pattern becomes gradually weaker until complete disappearance, even though the morphology remains the same. These hardly resolved patterns indicate that a poor texture or weak crystallinity layer forms at the surface of thicker region. However, a strong biaxial texture in the homogeneous zone is still detected by the conventional $\theta-2 \theta$ scans and texture measurements. It is deduced that homogeneous nucleation in the film body coincides with heterogeneous nucleation, i.e., epitaxial grains forming close to the interface contribute to the apparent sharp texture of the bulk, while polycrystalline grains with weak crystallinity form at the surface.

In order to further interpret this texture variation on film surface, we considered the amount of carbon residue, which is
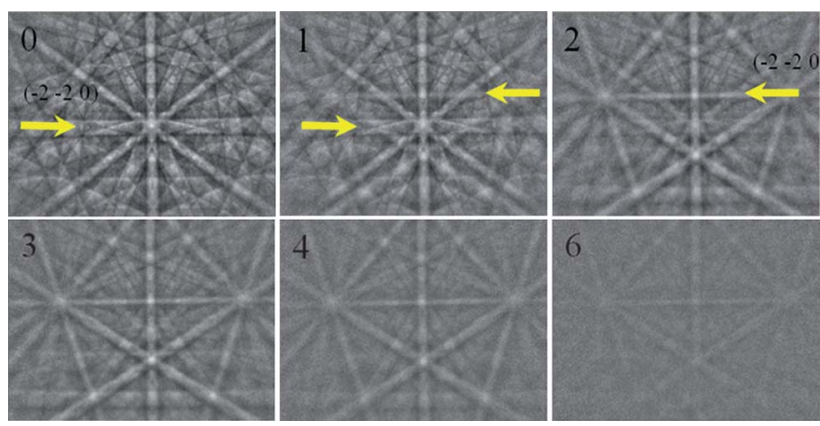

Fig. 2 Kikuchi patterns acquired at different positions numbered from 0 to 6 in Fig. 1(a). Strong reflections $(\overline{2} \overline{2} 0)$ of Ni as well as $(\overline{2} \overline{2} 0)$ of $\mathrm{Gd}_{2} \mathrm{Zr}_{2} \mathrm{O}_{7}$ are marked in the pattern 0 and 2, respectively. one of the main issues in chemical solution derived film crystallized in reducing atmosphere. ${ }^{20,29,30}$ XPS surface analysis reveals the amount of carbon residue in the homogeneous zone of films quenched from different stages during crystallization, as shown in Fig.3a. Although $\mathrm{CO}_{2}$ adsorption has influence on the absolute intensity of the $\mathrm{C} 1 \mathrm{~s}$ peak, it can still be clearly concluded that the evolution of the amount of aliphatic carbon residue is processing dependent, i.e., the amount of carbon decreases with the crystallization proceeding. Additionally, the trend of carbon amount changing from the surface to the body of the film by $\mathrm{C} 1 \mathrm{~s}$ depth analysis also provides a clue to carbon evolution in the sample surface layer during crystallization in the reducing atmosphere (Fig.3b). The films quenched from the beginning (initial) and the end (final) of the crystallization show respectively the highest and the lowest amount of carbon in the detected layers at all thicknesses, and the carbon amount almost remains constant over the investigated thickness range (except in the surface layer). Secondly, the carbon amount decreases gradually from the surface into the bulk in the intermediate state sample, revealing that the carbon elimination is a diffusion related process from the bottom of the film to its top surface. One should also note that highly crystallized grains with a strong texture can still be achieved at the same conditions in the non-fully covered zones, e.g. from zones I to III. Therefore, the carbon elimination accompanies the amorphous to crystallization transition in the thin films. As a conclusion, as the film thickness increases, the carbon elimination becomes difficult, and carbon segregation at the surface layer further suppresses epitaxial grain growth throughout the thickness in thick films. That is the reason why
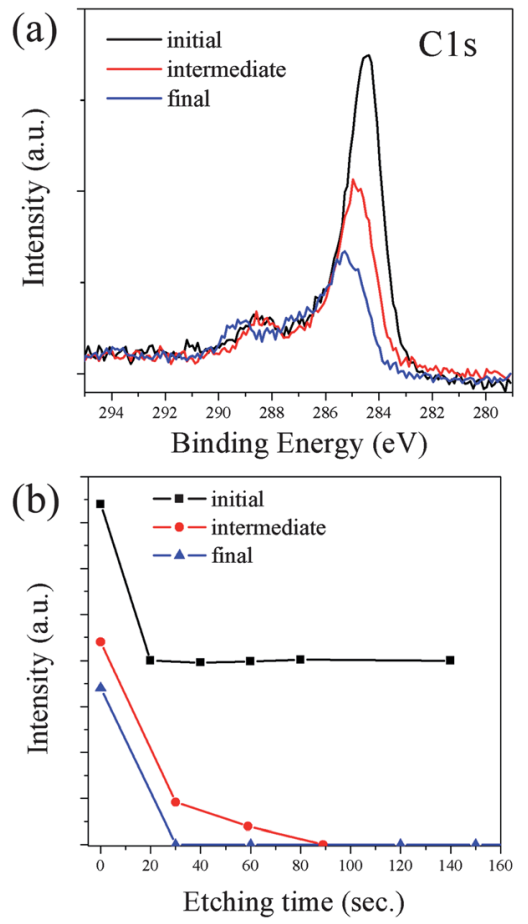

Fig. 3 XPS surface analysis (a) and the depth profile (b) of the surface layers in the homogeneous zone of samples quenched at different stages. "Initial", "intermediate" and "final" represent samples quenched from $900{ }^{\circ} \mathrm{C}, 1000{ }^{\circ} \mathrm{C}$ without any dwell and $1000{ }^{\circ} \mathrm{C}$ with $30 \mathrm{~min}$ dwell, respectively. All the samples were coated under the same conditions, and crystallized and quenched in a strongly reducing atmosphere. 
a strong dependence of surface texture on film thickness is observed in the fully covered region shown in Fig. 1a.

\section{Effect of $\mathrm{PO}_{2}$ on formation of $\mathrm{Gd}_{2} \mathrm{Zr}_{2} \mathrm{O}_{7}$ thin films}

According to the above results, we know that aliphatic carbon residue is one of the key issues responsible for surface texture formation in relatively thick films. Raising annealing temperature or prolonging the dwell time facilitates the elimination of carbon; however, both of these two approaches have limits, such as accelerating the diffusion of substrate elements or increasing production costs. Adding extra oxygen in the processing atmosphere during crystallization or post-annealing stage is an effective solution to eliminate the carbon residues under low temperature in a short time. ${ }^{20,29}$ Nevertheless, the influence of atmosphere with various $\mathrm{PO}_{2}$ on oxide films deposited on metallic substrates is more complicated than those on single crystals. First, the stability of the NiW substrate in various $\mathrm{PO}_{2}$ at a given crystallization temperature must be taken into consideration. According to the stability diagram for NiW alloy reported by $\mathrm{K}$. J. Leonard et al. ${ }^{31}$ it is known that oxides (i.e. $\mathrm{NiWO}_{4}, \mathrm{WO}_{2}$, etc.) form when the $\mathrm{PO}_{2}$ is higher than $10^{-13} \mathrm{~atm}$ at $1000{ }^{\circ} \mathrm{C}$. We thus selected five different $\mathrm{PO}_{2}$ values below the stability line for the annealing atmospheres, as shown in Fig. 4a.

Fig.4b presents the $\theta-2 \theta$ scans of the GZO films prepared in the atmospheres with various $\mathrm{PO}_{2}$. There is neither a significant influence of $\mathrm{PO}_{2}$ on GZO phase formation nor any impurity detected. The lattice constant of the GZO films crystallized under various atmospheres remains the same as well. However, a pronounced difference in terms of texture formation is found. A strong $c$-axis texture forms in the film processed in low $\mathrm{PO}_{2}(\mathrm{~A}$ or $\mathrm{B})$ atmospheres. When the $\mathrm{PO}_{2}$ is higher than $10^{-17} \mathrm{~atm}$, the films tend to exhibit evident polycrystalline orientation, where the intensities of the GZO (222) and (004) reflections are equivalent. Such an effect is much clearer when using $\mathrm{PO}_{2}$ of $10^{-16} \mathrm{~atm}$. Furthermore, undesirable oxides form under the conditions where the $\mathrm{PO}_{2}$ is higher than $10^{-15}$ atm.

The morphologies of these films are also shown in Fig. 5. Similar to the XRD results, there is no obvious difference between the samples A and B, both of which consisting of nanograins with an average grain size of about $10 \mathrm{~nm}$, as well as a small amount of pinholes. Using higher $\mathrm{PO}_{2}$, the films have larger grain size and tend to become rough. Dome-like grains with a grain size of $15 \mathrm{~nm}$ are clearly observed in sample D. Combining the XRD and SEM results, it is interesting to notice that the weakest intensity is detected in sample D, which has well developed grains. A possible explanation for this is that some minor reaction occurs between the GZO film and the NiW substrate at high $\mathrm{PO}_{2}$. The impurities that form during the reaction may consume some amount of the GZO precursors or films, leading to weaker reflection intensities in the well crystallized sample D. Similar reactions at the film-substrate interface were observed in other oxide films under reducing atmosphere, even using single crystals as substrates. ${ }^{19,20,32,33}$

\section{Proposal of a two-step annealing procedure for GZO films grown on Ni5W substrates}

On the basis of the above discussion, we know that low $\mathrm{PO}_{2}$ is favorable for keeping a "clean interface", thus, desirable epitaxial

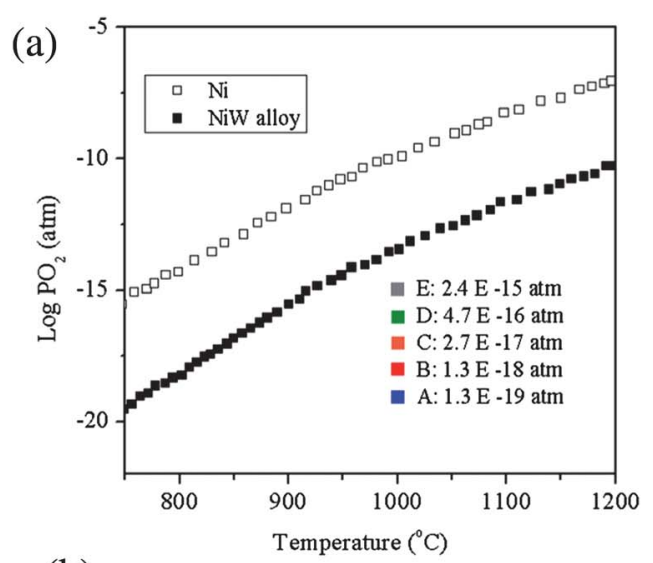

(b)

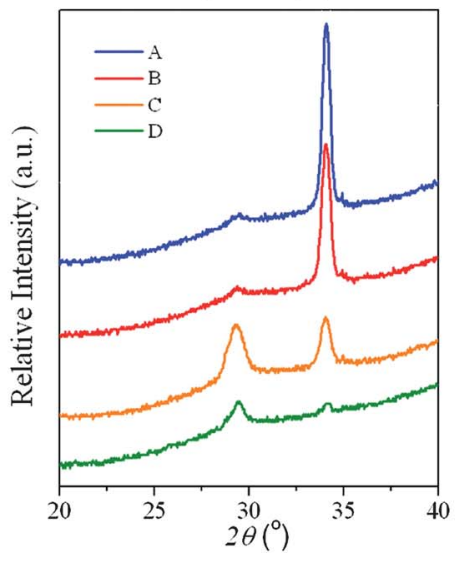

Fig. 4 (a) Stability diagram of the Ni5W alloy, showing the five $\mathrm{PO}_{2}$ values of the reducing atmospheres selected under the stability line and used for crystallizing the films at $1000{ }^{\circ} \mathrm{C}$, (b) XRD patterns of the $\mathrm{Gd}_{2} \mathrm{Zr}_{2} \mathrm{O}_{7}$ films crystallized in atmospheres with various $\mathrm{PO}_{2}$.

nucleation can be achieved. Inevitably, the carbon residue segregates in the film surface, which suppresses further epitaxial grain growth. We therefore propose a two-step annealing process (TSA, the profile of which is shown in Fig. 6.) for GZO films grown on technical substrates, which has been successfully used in other oxide films deposited on single crystals in ref. 20. During the first stage, a strongly reducing atmosphere with low $\mathrm{PO}_{2}$ is used and a fully covered GZO crystalline layer with good orientation is expected to

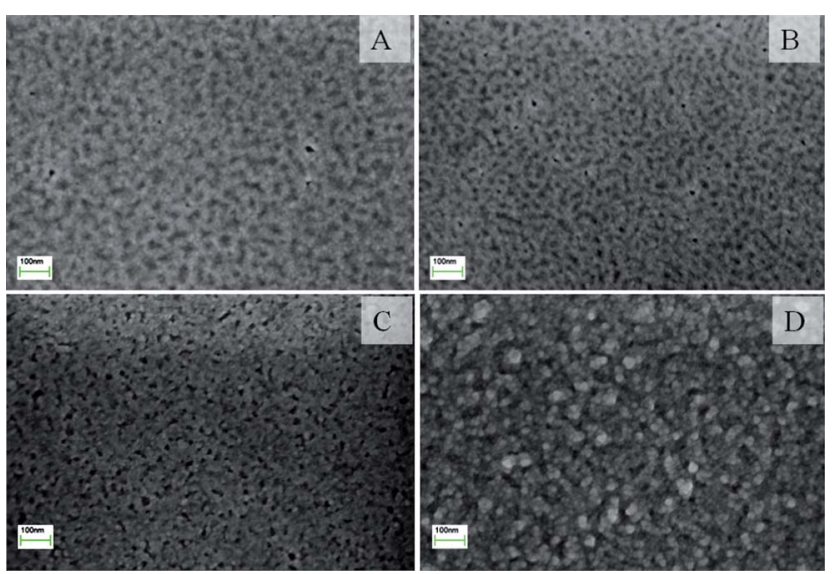

Fig. 5 SEM images of the $\mathrm{Gd}_{2} \mathrm{Zr}_{2} \mathrm{O}_{7}$ films crystallized in atmospheres with various $\mathrm{PO}_{2}$. 


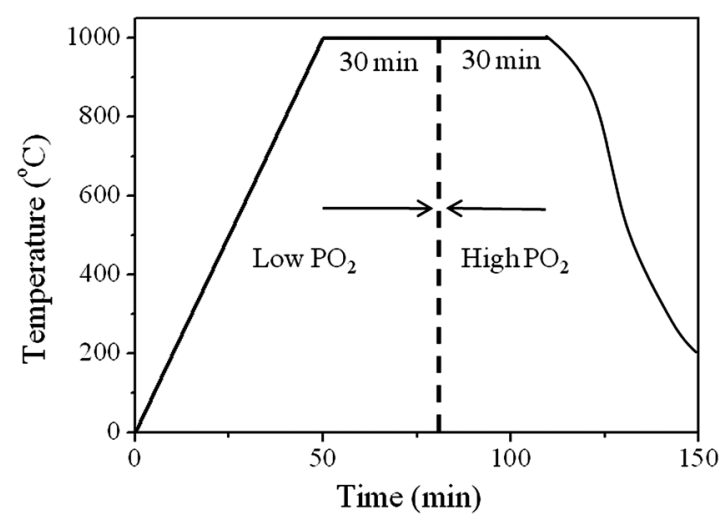

Fig. 6 Schematic procedure of the two-step heat treatment profile.
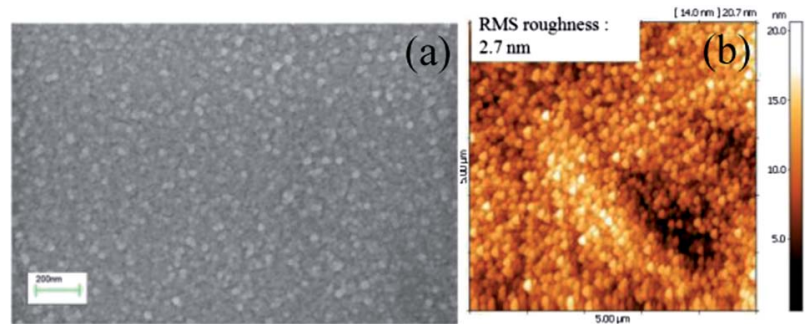

Fig. 7 Morphology of a $\mathrm{Gd}_{2} \mathrm{Zr}_{2} \mathrm{O}_{7}$ film prepared by a two-step annealing procedure observed by (a) SEM, and (b) AFM.

form close to the interface. During the second stage, an atmosphere with higher $\mathrm{PO}_{2}$ is used for improving the surface texture by further epitaxial grain growth from the bottom to the top.

The film prepared by the TSA procedure and the another prepared by the conventional route show similar $\theta-2 \theta$ scan patterns, indicating that no substrate oxidation occurs during annealing at higher $\mathrm{PO}_{2}$ atmosphere (also confirmed by the TEM cross-sectional image in Fig. 9b). That is due to the excellent oxygen barrier behavior of the GZO material. However,
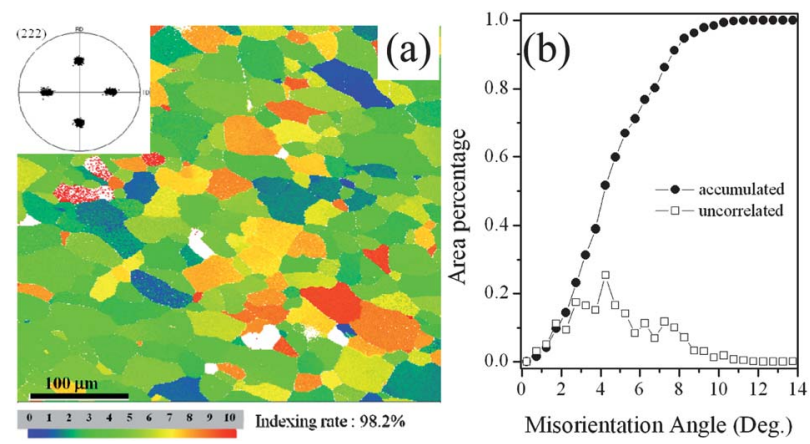

Fig. 8 EBSD data acquired from the $\mathrm{Gd}_{2} \mathrm{Zr}_{2} \mathrm{O}_{7}$ film prepared by the two-step heat treatment procedure. (a) Misorientation map, where the (222) pole figure calculated from this region is inserted in the left top corner. Highlighted colours (from blue to red in the colour bar) represent tolerance angles deviating from the ideal orientation from $0^{\circ}$ to $10^{\circ}$, while zero solution points are present as white; (b) misorientation distribution. The uncorrelated curve represents the distribution of relative misorientation between points in the sample, while the accumulated curve, which is integrated from the uncorrelated curve, reflects the area percentage variation of a certain texture component (i.e. GZO [110](001) in this case) as a function of the tolerance angle. significant changes have been observed in the SEM images, i.e. the mean grain size increases from $10 \mathrm{~nm}$ to $22 \mathrm{~nm}$ when using the TSA procedure, while the pinhole defects resulting from the released gas almost disappear. That suggests that an enhanced densification process takes place in parallel with further grain growth, thanks to the effective removal of carbon resulting from the higher oxygen content in the atmosphere. The low roughness value obtained from the AFM image (Fig.7b) also indicates high surface flatness achieved in the TSA film. Most importantly, an EBSD map with a high indexing rate of about $98.2 \%$ is obtained on the surface of the GZO film (Fig. 8a). The well defined granular structure with texture inherited from the NiW substrate underneath is observed. The zero solution points (marked as white spots in the map) mostly concentrate in the GB regions of the NiW substrate and are due to the overlapping Kikuchi patterns from several nanosize grains with relatively large
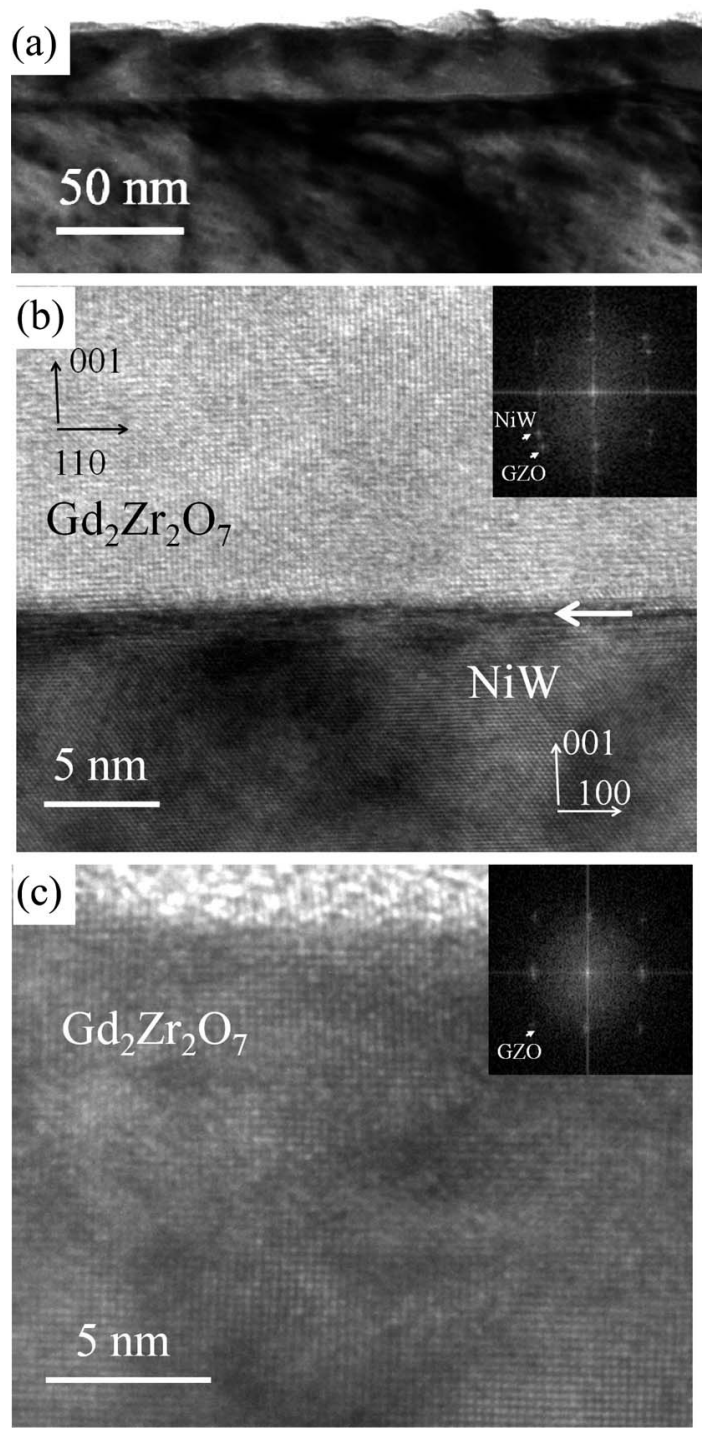

Fig. 9 (a) Low magnification cross-sectional TEM images of a $\mathrm{Gd}_{2} \mathrm{Zr}_{2} \mathrm{O}_{7}$ film prepared by the two-step annealing procedure. Zoom in (b) at the $\mathrm{Gd}_{2} \mathrm{Zr}_{2} \mathrm{O}_{7}-\mathrm{NiW}$ interface, (c) at the $\mathrm{Gd}_{2} \mathrm{Zr}_{2} \mathrm{O}_{7}$ surface layer, and insets are fast Fourier transform spectra acquired from area (b) and (c), respectively. 
misorientation angles. By analyzing the misorientation distribution curves plotted in Fig. 8b, we found a concentrated distribution at small misorientation angles ranging from $2^{\circ}$ to $7^{\circ}$ (uncorrelated curve) and the low tolerance angle of $8^{\circ}$ for saturated area percentage (accumulated curve), both of which are similar to the values of NiW substrate. TEM cross-sectional images of the GZO film are also shown in Fig. 9a, where a dense, pinhole free structure without significant thickness variation is observed. The sharp interface proves that no impurity forms at the second stage during TSA procedure. Both the regions close to the interface and at the surface layer (Fig. 9b and c) exhibit highly ordered crystallographic alignment with respect to that of the NiW substrate, as evidenced by the concentrated spots of the GZO lattice and NiW lattice in the fast Fourier transform spectrum. All those results offer strong evidence that after effective carbon elimination during the second step in the annealing procedure, epitaxial grains with growth advantage could further develop by consuming the polycrystalline grains in the surface layer, therefore, a significant improvement of the surface texture in GZO films can be achieved at a relatively low temperature in a short time.

\section{Conclusions}

In this work, the surface morphology and texture dependence on film thickness in GZO films deposited on NiW substrates by the chemical solution method are discussed, based on the detailed characterization of a region with thickness gradient in the crystallized GZO film by SEM and EBSD techniques. We found that the morphological features are highly dependent on the critical thickness, where the transition from FW growth mode to VW growth mode occurs. Highly ordered nanodots and networks with atomically flat top surfaces can be achieved by regulating the film thickness and the heat treatment conditions. Combined analysis of EBSD data on the film surface and XRD data from the bulk of the film shows that epitaxial grains form close to the interface, while polycrystalline grains are present on the surface. This is mainly due to the inhibition of epitaxial grain growth caused by the aliphatic carbon residue segregating in the surface layer. Study of the $\mathrm{PO}_{2}$ effect on texture formation in GZO films reveals that simply increasing the $\mathrm{PO}_{2}$ leads to polycrystalline texture throughout the film because of the chemical reactions between the substrate and the GZO layer, even though the grain growth is indeed promoted. Accordingly, a two-step heat treatment procedure for GZO films grown on technical substrates is developed. An atmosphere with a low $\mathrm{PO}_{2}$ is used at the initial stage to minimize the undesired reactions occurring at the interface, while a higher $\mathrm{PO}_{2}$ atmosphere is introduced during the following stage to promote further epitaxial grain growth. As a result, a highly textured GZO film with dense, pinhole free structure is obtained. This work provides a novel methodology to investigate the thickness effect on films derived by the chemical solution method, and also demonstrates the possibilities of engineering the surface morphology and texture of buffer layers grown directly on technical substrates.

\section{References}

1 A. Hörling, L. Hultman, M. Oden, J. Sjolen and L. Karlsson, Surf. Coat. Technol., 2005, 191, 384-392.

2 N. Y. Lee, J. R. Lim, M. J. Lee and S. Park, Chem. Mater., 2005, 17, $5867-5870$.
3 A. E. Pashitski, A. Gurevich, A. A. Polyanskii, D. C. Larbalestier, A. Goyal, E. D. Specht, D. M. Kroeger, J. A. DeLuca and J. E. Tkaczyk, Science, 1997, 275, 367-369.

4 M. W. Rupich, X. P. Li, C. Thieme, S. Sathyamurthy, S. Fleshler, D. Tucker, E. Thompson, J. Schreiber, J. Lynch, D. Buczek, K. DeMoranville, J. Inch, P. Cedrone and J. Slack, Supercond. Sci. Technol., 2010, 23, 014015 .

5 V. Selvamanickam, Y. Chen, I. Kesgin, A. Guevara, T. Shi, Y. Yao, Y. Qiao, Y. Zhang, G. Majkic, G. Carota, A. Rar, Y. Xie, J. Dackow, B. Maiorov, L. Civale, V. Braccini, J. Jaroszynski, A. Xu, D. C. Larbalestier and R. Bhattacharya, IEEE Trans. Appl. Supercond., 2011, 21, 3049-3054.

6 T. Izumi and Y. Shiohara, Phys. C, 2010, 470, 967-970.

7 C. Cantoni, D. K. Christen, R. Feenstra, D. P. Norton, A. Goyal, G. Ownby and D. M. Zehener, Appl. Phys. Lett., 2001, 79, 3077-3079.

8 A. Aytug, M. Paranthaman, K. J. Leonard, S. Kang, P. M. Martin, L. Heatherly, A. Goyal, A. O. Ijaduola, J. R. Thompson, D. K. Christen, R. Meng, I. Rusakova and C. W. Chu, Phys. Rev. B: Condens. Matter Mater. Phys., 2006, 74, 184505.

9 M. Sparing, E. Backen, T. Freudenberg, R. Hühne, B. Rellinghaus, L. Schultz and B. Holzapfel, Supercond. Sci. Technol., 2007, 20, S239-S246.

10 M. Coll, J. Gazquez, R. Hühne, B. Holzapfel, Y. Morilla, J. GarciaLopez, A. Pomar, F. Sandiumenge, T. Puig and X. Obradors, J. Mater. Res., 2009, 24, 1446-1455.

11 Y. F. Qiao, Y. M. Chen, X. M. Xiong, S. J. Kim, V. Matias, C. Sheehan, Y. Zhang and V. Selvamanickam, IEEE Trans. Appl. Supercond., 2011, 21, 3055-3058.

12 V. Mihalache and I. Pasuk, Acta Mater., 2011, 59, 4875-4885.

13 K. Develos-Bagarinao, H. Yamasaki and Y. Nakagawa, Supercond. Sci. Technol., 2006, 19, 873-882.

14 R. Lo Nigro, R. G. Toro, G. Malandrino and I. L. Fragala, J. Mater. Chem., 2005, 15, 2328-2337.

15 F. F. Lange, Science, 1996, 273, 903-909.

16 R. W. Schwartz, Chem. Mater., 1997, 9, 2325-2340.

17 M. S. Byuiyan, M. Paranthaman and K. Salama, Supercond. Sci. Technol., 2006, 19, R1-R21.

18 S. Sathyamurthy, K. Kim, T. Aytug and M. Paranthaman, Chem. Mater., 2006, 18, 5829-5831.

19 K. Venkataraman and E. Hellstrom, J. Mater. Res., 2009, 24, 1567-1575.

20 A. Guibadj, P. Odier, B. Benbarta and L. Ortega, J. Cryst. Growth, 2011, 328, 102-107.

21 Y. Zhao, J. C. Grivel, D. He, P. G. P. A. Pallewatta, A. B. Abrahamsen, J. Bednarík and M. v. Zimmermann, submitted to Physics Procedia.

22 Z. M. Yu, P. Odier, S. Morlens, P. Chaudouet, M. Bacia, L. Zhou, P. X. Zhang, L. H. Jin, C. S. Li, P. David, O. Fruchart and Y. F. Lu, J. Sol-Gel Sci. Technol., 2010, 54, 363-370.

23 J. K. Brendan, Q. D. Zhou and A. Maxim, J. Solid State Chem., 2011, 184, 1695-1698.

24 Y. X. Zhou, X. Zhang, F. Fang, P. T. Putman and K. Salama, IEEE Trans. Appl. Supercond., 2007, 15, 2711-2714.

25 T. Aytug, M. Paranthaman, K. J. Leonard, H. Y. Zhai, M. S. Bhuiyan, E. A. Payzant, A. Goyal, S. Sathyamurthy, D. B. Beach, P. M. Martin, D. K. Christen, X. Li, T. Kodenkandath, U. Schoop, M. W. Rupich, H. E. Smith, T. Haugan and P. N. Barnes, J. Mater. Res., 2005, 20, 2988-2996.

26 Y. Zhao, J. C. Grivel, M. Napari, D. Pavlopoulos, J. Bednarcik and M. Von Zimmermann, Thin Solid Films, 2012, 520, 1965-1972.

27 Y. H. Chen, X. L. Ye and Z. G. G. Wang, Nanoscale Res. Lett., 2006, 1, 79-83.

28 J. A. Venables, G. D. T. Spiller and M. Hanbucken, Rep. Prog. Phys., 1984, 47, 399-459.

29 A. Cavallaro, F. Sandiumenge, J. Gàzquez, T. Puig, X. Obradors, J. Arbiol and H. C. Freyhardt, Adv. Funct. Mater., 2006, 16, 13631372 .

30 M. Coll, J. Gázquez, J. Sandiumenge, T. Puig, X. Obradors, J. P. Espinós and R. Hühne, Nanotechnology, 2008, 19, 395601.

31 K. J. Leonard, A. Goyal, S. Kang, K. A. Yarborough and D. M. Kroeger, Supercond. Sci. Technol., 2004, 17, 1295-1302.

32 L. Molina, H. Y. Tan, E. Biermans, K. J. Batenburg, J. Verbeeck, S. Bals and G. V. Tendeloo, Supercond. Sci. Technol., 2011, 24, 065019.

33 S. Petit, M. Mikolajczyk, J.-L. Soubeyroux, T. Waeckerle, R. Batonnet, S. Pairis and P. Odier, IEEE Trans. Appl. Supercond, 2011, 21, 2977-2980. 